\title{
(0) \\ Conocimiento matemático de estudiantes para docentes de Educación Primaria: Análisis de variables
}

\author{
Mathematical knowledge of elementary education student \\ teachers: variable analysis
}

Conhecimento matemático de estudantes para docentes do Ensino

Fundamental: Análise de variáveis

Jaime Segarra $^{1}$ Carmen Julià ${ }^{1}$

Received: Mar/18/2020 • Accepted: Apr/28/2020 • Published: Jan/31/2021

\section{Resumen}

En este trabajo investigativo, se estudia el conocimiento de números y geometría de estudiantes para docentes de Educación Primaria. La investigación es de tipo cuantitativo y se utilizaron técnicas de estadística descriptiva e inferencial. Para abordar el estudio, se seleccionaron 20 preguntas liberadas de las pruebas TIMSS y se aplicaron a 97 estudiantes de primer año del grado de Educación Primaria. Los resultados se presentaron en dos secciones diferentes. La primera de ellas muestra que quienes provienen de un bachillerato de Ciencias y quienes recibieron formación matemática en los dos últimos años obtuvieron puntuaciones más altas $(p<0.05)$. En la segunda sección, se indica que el estudiantado obtiene puntuaciones más altas en el dominio de contenidos de números y en el dominio cognitivo de conocer $(p<0.05)$. La indagación permitirá reforzar las debilidades que presentan los grupos de aprendices al rediseñar la asignatura Enseñanza y Aprendizaje de Matemáticas.

Palabras clave: Aritmética; geometría; profesores en formación; Educación Primaria; razonamiento; TIMSS; educación matemática; conocimiento matemático

\section{Abstract}

The paper studies the knowledge elementary school student teachers have regarding numbers and geometry. This research study is quantitative and used descriptive and inferential statistical techniques. A total of 20 released questions were selected from the TIMSS test and applied to 97 first-year students in an Elementary Education program. Results were presented in two different sections. The first section shows that those who come from a Bachelor's Degree in Science and have received mathematics in the last two years obtained higher scores $(p<0.05)$. The second section indicates that students obtain higher scores in the number content domain and the knowing cognitive domain $(p<0.05)$. The study will strengthen the weaknesses presented by the groups of students when redesigning the Teaching and Learning Mathematics course.

Jaime Segarra, $\$ jaimerodrigo.segarra@urv.cat. ㄱ https://orcid.org/0000-0003-4304-2385

Carmen Julià, $\$ carme.julia@urv.cat. (1) https://orcid.org/0000-0003-3440-6175

1 Departamento de Ingeniería Informática y Matemáticas, Universitat Rovira i Virgili, Tarragona, España. 
Keywords: Arithmetic; geometry; teachers in training; elementary education; reasoning; TIMSS; Mathematics education; Mathematical knowledge

\section{Resumo}

Neste trabalho investigativo, é estudado o conhecimento de números e geometria de estudantes para docentes de Educação Primária. A pesquisa é quantitativa e foram utilizadas técnicas de estadística descritiva e inferencial. Para abordar o estudo, foram selecionadas 20 perguntas disponíveis dos testes TIMSS e aplicadas a 97 estudantes do primeiro ano do Ensino Fundamental. Os resultados foram apresentados em duas seções diferentes. A primeira delas expõe que aqueles que provêm de um bacharelato em Ciências e aqueles que receberam formação matemática nos dois últimos anos obtiveram pontuações mais altas $(p<0,05)$. Na segunda seção, indica que o corpo discente obtém pontuações mais altas no domínio de conteúdos de números e no domínio cognitivo de conhecimento $(p<0,05)$. A questão permitirá reforçar as debilidades apresentadas pelos grupos de aprendizes ao redesenhar a matéria de Ensino e Aprendizagem da Matemática.

Palavras-chave: Aritmética; geometria; professores em formação; Ensino Fundamental; raciocínio; TIMSS; Educação matemática; Conhecimentos matemáticos

\section{INTRODUCCIÓN}

El personal docente de primaria tiene un rol muy importante en la educación, ya que se considera que influyen directamente en el aprendizaje de sus estudiantes, por lo que es necesario realizar una investigación desde su formación inicial, con el propósito de mejorar el aprendizaje del conocimiento matemático de enseñantes para el futuro. $\mathrm{Ma}$ (2010) sostuvo que gran parte del éxito en el aula depende de la propia comprensión de la matemática del profesor o profesora acerca de ella, sumado a una adecuada apropiación disciplinar, y a una seguridad y autoconfianza en el dominio de dicho conocimiento. En este sentido, Haciomeroglu (2006) afirmó que docentes con un fuerte conocimiento matemático tienen mejor preparación para ayudar a sus estudiantes a comprender significativamente el tema.

Por otra parte, el hecho de querer conocer el nivel del conocimiento matemático del estudiantado al iniciar la Educación
Primaria se da con el propósito de reforzar los contenidos en los que presentan dificultades. Diversas investigaciones resaltaron la importancia de que el personal docente posea una buena preparación matemática para ser más efectivo al momento de enseñar la materia (Flores et al., 2018). Además, Martín del Pozo et ál. (2013), en su estudio, mostraron que la mayoría de maestras y maestros entrevistados declara que es muy importante dominar los contenidos para poderlos enseñar correctamente. El conocimiento profesional que debe tener el profesorado es determinante para la efectividad de la enseñanza (Mahler et al., 2017). Sin duda, los futuros cuerpos docentes deben poseer una excelente formación inicial que asegure conocimientos sólidos en contenidos y didácticos, para que en el futuro sean profesionales de calidad.

Para que la formación inicial de docentes de primaria sea más efectiva, es necesario tener en cuenta los conocimientos que deben poseer, por lo que se revisa el planteamiento de Shulman (1987), quien 
consideró que el conocimiento matemático para la enseñanza tiene dos componentes: el conocimiento del contenido matemático y el conocimiento didáctico del contenido matemático. Para determinar el conocimiento matemático para la enseñanza, el equipo de investigación que presenta este artículo aplicó diferentes pruebas de índole nacional e internacional, lo cual se muestra en la revisión de la bibliografía.

Para llevar a cabo la investigación, se plantean diversas hipótesis, que tienen en cuenta cada una de las variables que se pretende analizar. En particular, se plantean cuatro hipótesis, separadas en dos apartados diferentes:

Análisis por el tipo de bachillerato y el tiempo de no estudiar matemática:

H1. Esta primera hipótesis corresponde a la variable: Tipo de bachillerato que estudiaron. Ho: "No existe diferencias significativas entre las puntuaciones de las medias de estudiantado graduado en Bachillerato de Ciencias con el de $\mathrm{Hu}-$ manidades y Ciencias Sociales".

H2. En este caso, se comparan los resultados, según si estudiantes que han recibido formación matemática en los dos últimos años. Ho: "No hay diferencias significativas en los resultados de las medias de estudiantes que no han estudiado la asignatura de Matemáticas en los últimos dos años y los que la habían estudiado durante estos dos últimos años".

Análisis por dominio de contenidos y dominio cognitivo:

H3. En este caso se estudia, si los resultados obtenidos dependen del dominio de contenido de las preguntas. Ho: "No hay diferencias significativas entre la media de las puntuaciones de las preguntas correspondientes al dominio de contenidos de números y las correspondientes a geometría".

H4. En esta última hipótesis, se estudia, si los resultados varían según el tipo de dominio cognitivo de las preguntas. Ho: "No existen diferencias significativas entre la media de las puntuaciones obtenidas en las preguntas correspondientes a cada dominio cognitivo (conocer, analizar y razonar)".

\section{REVISIÓN BIBLIOGRÁFICA}

El estudio más importante a nivel internacional en estudiantes a maestro o maestra fueron las pruebas TEDM-S (Teacher Education Study in Mathematics). El estudio comparativo TEDM-S se llevó a cabo durante los años 2006-2010 para docentes de educación primaria y secundaria en formación, con la participación de 17 países. España participó en el estudio y obtuvo 481 puntos en conocimientos matemáticos, por debajo de la media (500 puntos), evidenciando deficiencias en los conocimientos de contenidos matemáticos y los conocimientos de la didáctica, lo que pone en alerta la preparación de la formación inicial de estos grupos profesionales.

Por otro lado, los estudios más importantes a nivel internacional en estudiantes de primaria y secundaria son el PISA (Programme for International Student Assessment) y el TIMSS (Third International Mathematics and Science Study). En la mayoría de los países que se aplica este tipo de pruebas se ha puesto en manifiesto las deficiencias de conocimiento matemático de estudiantes de primaria y secundaria. Martín y Puertas (2018) afirmaron que la presión política para evaluar el rendimiento de estudiantes escolares ha provocado la existencia 
de sistemas de evaluación educativa que aporten periódicamente información sobre la situación internacional de los procesos de aprendizaje. Estos estudios permiten realizar comparaciones entre países y se utilizan como una medida relativa de la calidad global existente.

El proyecto TIMSS evalúa el rendimiento de estudiantes en matemáticas y ciencias, cuantificando el alcance del aprendizaje en estas dos materias, así como del contexto en el que ocurre (Martín \& Puertas, 2018). TIMSS evalúa el conocimiento del contenido matemático en cuatro dominios: números, álgebra, geometría, y datos y probabilidad. Cada uno de estos dominios presenta objetivos y destrezas o habilidades. Por otra parte, las preguntas se clasifican según los dominios cognitivos conocer, aplicar y razonar.

En TIMSS 2011, estudiantes de Educación Primaria de España consiguieron 482 puntos, por debajo de la media de la OCDE (Organización para la Cooperación y el Desarrollo Económico) (522). En TIMSS 2015, España alcanzó 505 puntos, por debajo de la media de la OCDE (525). Según Gutiérrez et ál. (2016), estos bajos resultados están relacionados con la deficiente formación docente, tanto en conocimientos matemáticos como didácticos.

En los últimos años se han publicado diversas investigaciones referentes a la formación inicial de maestros y maestras, a continuación, se revisan algunos de estos estudios.

En su estudio, Turnuklu y Yesildere (2007) investigaron el conocimiento de las matemáticas y el conocimiento de la enseñanza de las matemáticas de docentes en formación. Los autores encontraron una conexión entre el conocimiento de las matemáticas y el conocimiento de la enseñanza de las matemáticas. Sugieren que los candidatos a docentes de matemática de primaria se eduquen tanto en los aspectos de conocimiento matemático como de conocimiento de contenido pedagógico.

En Nortes y Nortes (2017a), los resultados indicaron que uno de cada tres estudiantes del Grado de Educación Primaria no posee competencia matemática de $6^{\circ} \mathrm{de}$ Primaria, en las preguntas de razonar y reflexionar el $50 \%$ de estudiantes suspenden, en las preguntas de aplicar y analizar el 20 $\%$ y en conocer y reproducir el $10 \%$. En todas los variables, los mejores resultados los obtienen los hombres y la geometría es el bloque con peores puntuaciones.

Giaconi et ál. (2018), en su investigación sobre prácticas docentes, autoeficacia y valor en relación con la resolución de problemas de matemática, mostraron que los profesorados de primaria y secundaria tienen la intención de trabajar la resolución de problemas no rutinarios, se sienten capaces para hacerlo y valoran este tipo de enseñanza; sin embargo, existen barreras que pueden ser individuales o externas que les dificultan su implementación.

En otro estudio, Nortes y Nortes (2018) realizaron una investigación de conocimientos matemáticos de futuros maestros y maestras en la resolución de problemas, aplicando la prueba "Evaluación final de competencia matemática de $6^{\circ}$ de Educación Primaria de la comunidad de Madrid". Los resultados indicaron que uno de cada tres estudiantes no supera las dos evaluaciones aplicadas y que en segundo (primer año con matemáticas) una de las evaluaciones no supera el 50 $\%$. Del total de participantes, tres de cada veinte estudiantes contestan bien todas las cuestiones. En general, presentaron la mayor dificultad en la equivalencia entre 
fracción, decimal y porcentaje, en donde tan solo uno de cada cuatro estudiantes contesta correctamente.

En una investigación más reciente, Alpízar y Alfaro (2019) investigaron sobre la formación universitaria de docentes de educación primaria. Realizaron una descripción de los programas de formación ofrecidos por algunas universidades estatales. Encontraron una amplia variedad de programas para formar docentes de educación primaria, sin lineamientos generales y poco control. Estos resultados resultan importantes porque podrían afectar la enseñanza de matemáticas en las instituciones de educación primaria, ya que llegan docentes con énfasis distintos en su formación.

Teniendo en cuenta que la formación inicial del profesorado es un elemento clave para la mejora de la calidad educativa (Muñoz et al., 2019), el objetivo principal de esta investigación es analizar el conocimiento del contenido matemático que el estudiantado posee al iniciar el Grado de Educación Primaria. La idea es detectar carencias o dificultades que presentan los futuros maestros o maestras e intentar superarlas. La investigación consiste en aplicar una prueba tipo TIMSS y, por un lado, analizar el conocimiento de los dominios de contenido de numeración y geometría, y los dominios cognitivos de conocimiento, aplicación y razonamiento. Por otro lado, analizar las variables tipo bachillerato que estudiaron y años sin recibir formación de matemáticas.

\section{METODOLOGÍA}

Esta investigación se puede clasificar como cuantitativa. Además, en este estudio, para el análisis de la información, se utiliza la estadística descriptiva e inferencial, con el propósito de detallar las características del conjunto de datos. La muestra utilizada en esta investigación es una muestra intencional (Patton, 2002). Concretamente, la población objeto de estudio corresponde a estudiantes de primer año del Grado de Educación Primaria de la Universitat Rovira i Virgili de España-Tarragona en el curso 2018/2019. Para la muestra se procedió a aplicar la prueba a todo el estudiantado que asistió el primer día de clase. La prueba se aplicó a un total de 97 estudiantes, que representan el $71 \%$ de la población total matriculada en primer año. En este estudio, el $57 \%$ son mujeres y el $43 \%$ son varones. Es importante destacar que el estudiantado del Grado de Educación Primaria no está cursando una formación específica de docente de matemáticas, sino que reciben la misma formación de esta materia, de forma obligatoria.

\section{Instrumento}

El instrumento que se usó para llevar a cabo la investigación recoge información de los conocimientos de los contenidos matemáticos: números y geometría. Se seleccionaron estos dos dominios de contenido, pues estudios previos han señalado la importancia del aprendizaje de la aritmética (números) en la formación matemática de los futuros grupos de docentes (Castro et ál., 2015; Nortes \& Nortes, 2017c) y la importancia de la geometría (Torregrosa et ál., 2010; Nortes \& Nortes, 2017b).

Medir el conocimiento del profesorado es una tarea compleja y existe poco consenso sobre la manera en que se debería realizar (Ball et ál., 2001). Por lo que, este trabajo investigativo trabaja con enunciados internacionales. La prueba se preparó a partir de las preguntas TIMSS 2011. Los esquemas y los procedimientos 
del estudio TIMSS se consideran como válidos para evaluar el conocimiento matemático (Gutiérrez et ál., 2016). De la prueba TIMMS se ha considerado el dominio de contenidos matemáticos de segundo de educación secundaria obligatoria (ESO), se seleccionaron 20 preguntas: 12 preguntas del dominio de contenido de números (P1-P12), que representa el $60 \%$ de la prueba, y 8 preguntas del dominio de contenido de geometría (P13-P20), que representan el $40 \%$. Once preguntas se plantearon para forzar que se evidenciara el proceso en el desarrollo de la respuesta y a estas nos referiremos como preguntas tipo problema (P5, P6, P7, P8, P9, P10, P11, P12, P17, P18, P19 y P20). Las demás preguntas mantienen la estructura de la prueba TIMSS, y nos referiremos a ellas como preguntas objetivas.

Los enunciados de las preguntas que conforman la prueba se presentan en la Tabla 1.1 del Anexo 1.

\section{Procedimientos y protocolo}

La prueba se aplicó al estudiantado el primer día de clase. Se dispuso de 40 minutos para contestar las 20 preguntas. Luego se procedió a calificar y a generar una base datos con toda la información que proporcionó esta prueba. Para la calificación se tuvo en cuenta lo siguiente: las preguntas objetivas puntúan $0 \mathrm{o} 1$, dependiendo de si son correctas o incorrectas. En el caso de las preguntas tipo problema, se tiene en cuenta el procedimiento además de la respuesta. Concretamente, la puntuación es 0 si la respuesta y el procedimiento son incorrectos, $0.5 \mathrm{si}$ la respuesta o el procedimiento son correctos y 1 si ambos son correctos. La prueba es calificada en una escala de 0 a 10 . Posteriormente, se realiza el análisis estadístico. Todos los cálculos de la estadística descriptiva e inferencial se realizaron mediante el lenguaje de programación R. Los gráficos se generaron en Microsoft Excel.

Se estudió la fiabilidad de la prueba mediante el cálculo del estadístico Alfa de Cronbach y se obtuvo un valor de $\alpha=0.791$, que corresponde a un nivel de fiabilidad satisfactorio (Hernández et ál., 2007).

\section{RESULTADOS}

Los resultados se presentan en dos apartados diferentes: 1) Análisis por el tipo de bachillerato y el tiempo de no estudiar matemática; 2) análisis del dominio de contenido y cognitivo.

\section{Análisis por el tipo de bachillerato y el tiempo de no estudiar matemática}

En este apartado se estudia el tipo de bachillerato estudiado por el estudiantado del Grado de Educación Primaria y el tiempo de no estudiar la asignatura de matemáticas.

Tipo de bachillerato estudiado por estudiantes del Grado de Educación Primaria (H1) En este, se comparan las puntuaciones obtenidas por estudiantes que cursaron el bachillerato de Ciencias con quienes cursaron el de Humanidades y Ciencias Sociales. En la muestra que se ha tomado para esta investigación, el $22 \%$ de estudiantes proviene del bachillerato de Ciencias, el $61 \%$ del bachillerato de Humanidades y Ciencias Sociales, el $4 \%$ del bachillerato de Artes y el $13 \%$ de otro tipo de estudios. En esta investigación se analizan los resultados de estudiantes provenientes del bachillerato de Ciencias y Humanidades y Ciencias Sociales. 
El Gráfico 1 muestra los diagramas de caja y bigotes de las puntuaciones totales obtenidas por estudiantes que provienen del bachillerato de Ciencias y quienes cursaron el de Humanidades y Ciencias Sociales $(H$. y Ciencias S.). Este tipo de diagrama permite identificar el primer y tercer cuartil, la mediana (línea horizontal) y la media (marcada con una cruz). Los bigotes se extienden desde la caja hasta los valores máximos y mínimos. En particular, se muestran tres diagramas: el correspondiente a las puntuaciones de toda la prueba (total), y, por separado, las puntuaciones correspondientes al dominio de números y las correspondientes al dominio de geometría.

También, en el gráfico 1 se puede observar que las puntuaciones obtenidas por estudiantado proveniente del bachillerato en Ciencias son claramente superiores al de $\mathrm{Hu}-$ manidades y Ciencias Sociales. En los resultados de la prueba que incluye los dos dominios de contenido matemático (total), el $25 \%$ de estudiantes provenientes de Ciencias (Q1) obtuvo notas inferiores a 5.44 y otro $25 \%$ (Q2) notas inferiores a 7.00. En el caso de estudiantes que provienen de Humanidades y Ciencias Sociales, un $25 \%$ de las notas son

inferiores a 4.75 (Q1) y un $50 \%$ inferior a 6.00 (Q2). Además, los dos tipos de bachillerato obtienen una distribución asimétrica negativa (es decir, en los dos casos existe mayor dispersión de datos en los valores por debajo de la mediana). Adicionalmente, se puede observar que la diferencia entre ambos grupos de bachillerato es similar en el caso de números y geometría.

Por otro lado, la Tabla 1 detalla la media y desviación estándar de las puntuaciones obtenidas considerando el total, el dominio de números y el de geometría. Se realizó un test estadístico para analizar si la diferencia entre las medias obtenidas en cada dominio es estadísticamente significativa. Concretamente, se aplicó el test estadístico t-Student, tomando el parámetro $\alpha=0.05$ con un nivel de significación del $5 \%$.

Teniendo en cuenta el valor de $\mathrm{p}$ que resulta del test estadístico $(\mathrm{p}=0.016)$, se rechaza la hipótesis nula (Ho) planteada en H1. También, se puede apreciar que hay diferencias significativas en las puntuaciones de la media por dominio de contenido matemáticos, en números $(\mathrm{p}=$ $0.017)$ y en geometría $(\mathrm{p}=0.049)$. Así, se puede afirmar que existen diferencias significativas entre

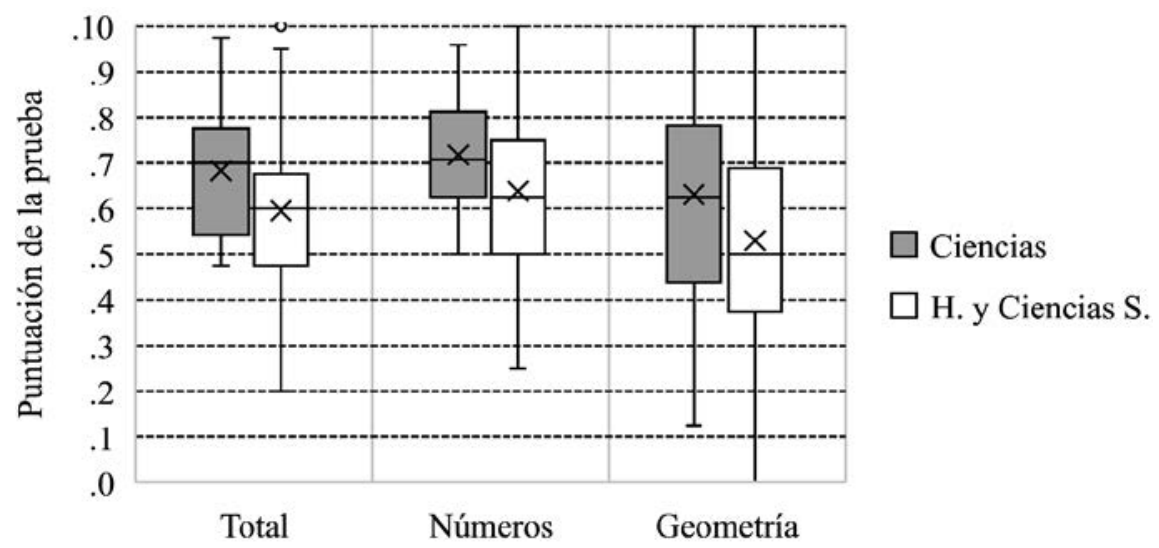

Gráfica 1. Puntuaciones totales y por dominio de contenido, según tipo de bachillerato

Nota: Fuente propia de la investigación. los resultados de estudiantes provenientes de los dos bachilleratos.

Tiempo de no estudiar la asignatura de matemáticas (H2). En este apartado se analiza la relación de los resultados correspondientes a estudiantes que han tenido la asignatura de matemáticas 
Tabla 1. Estadísticos de Ciencias y Humanidades y Ciencias Sociales

\begin{tabular}{lcccccc}
\hline & \multicolumn{2}{c}{ Total } & \multicolumn{2}{c}{ Números } & \multicolumn{2}{c}{ Geometría } \\
& Ciencias & H. y Ciencias S. & Ciencias & H. y Ciencias S. & Ciencias & H. y Ciencias S. \\
\hline Media & 6.83 & 5.94 & 7.17 & 6.38 & 6.30 & 5.29 \\
Desviación & 1.50 & 1.78 & 1.33 & 1.74 & 2.39 & 2.57 \\
p-valor & & 0.016 & \multicolumn{2}{c}{0.017} & \multicolumn{2}{c}{0.049} \\
\hline
\end{tabular}

Nota: Fuente propia de la investigación.

en los últimos dos años (39\% de la población) con estudiantes que no han estudiado matemáticas en los últimos dos años (61\% de la población).

El Gráfico 2 muestra el diagrama de caja y bigotes de los resultados correspondientes a estudiantes que han tenido la asignatura de matemáticas en los dos últimos años y quienes no la han tenido. Como en el caso anterior, se muestra la puntuación total y las puntuaciones por dominio de contenido matemático.

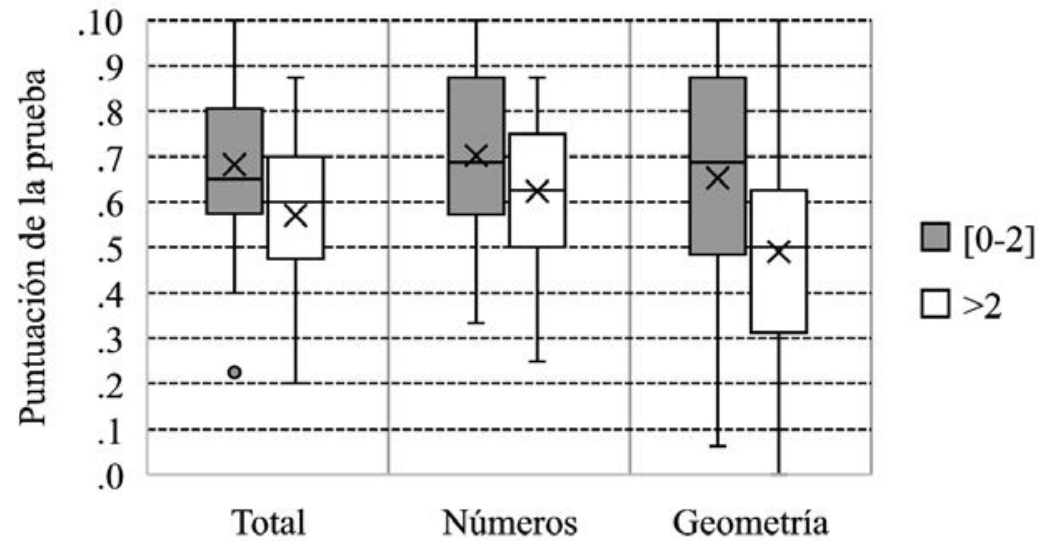

Gráfica 2. Puntuaciones totales y por dominio de contenido, según tiempo de no estudiar matemáticas Nota: Fuente propia de la investigación.

Tabla 2. Estadísticos de tiempo de no estudiar contenidos matemáticos

\begin{tabular}{|c|c|c|c|c|c|c|}
\hline & \multicolumn{2}{|c|}{ Total } & \multicolumn{2}{|c|}{ Números } & \multicolumn{2}{|c|}{ Geometría } \\
\hline & {$[0-2]$} & Más de 2 & {$[0-2]$} & Más de 2 & {$[0-2]$} & Más de 2 \\
\hline Media & 6.82 & 5.70 & 7.01 & 6.23 & 6.53 & 4.90 \\
\hline Desviación & 1.78 & 1.60 & 1.77 & 1.50 & 2.47 & 2.44 \\
\hline$\rho$-valor & 0.0012 & & 0.014 & & 0.0011 & \\
\hline
\end{tabular}

Nota: Fuente propia de la investigación.
El Gráfico 2 muestra que estudiantes que han estudiado en los dos últimos años matemáticas obtienen claramente puntaciones más altas. La mediana obtenida en la prueba por estos grupos de estudiantes es de 6.50, mientras que estudiantes que no han tenido matemáticas en los últimos dos años obtienen una mediana de 6.00. Por dominio de contenido matemático, los resultados más relevantes se dan en geometría, que obtiene en el primer y el segundo caso una mediana de 6.88 y 5.00 , respectivamente.

La Tabla 2 muestra la media y la desviación estándar obtenida por cada grupo (total, números y geometría) y el valor de p resultante de la aplicación del test estadístico t-Student.

Con un valor de $\mathrm{p}=$ 0.0012 , se rechaza la hipótesis nula (Ho) planteada en H2. Es decir, la diferencia entre las medias de las puntuaciones obtenidas es estadísticamente significativa mayor en estudiantes que han tenido matemáticas en los dos últimos años. Esta diferencia significativa se ve también en los dos dominios de contenido matemático: números $(\mathrm{p}=0.014) \mathrm{y}$ geometría $(\mathrm{p}=0.0011)$. 


\section{Análisis del dominio de contenido y del dominio cognitivo}

Dominio de contenido números y geometría (H3). En este apartado, se realiza una comparativa entre los resultados obtenidos en las preguntas correspondientes al dominio de contenido matemático de números y geometría.

En primer lugar, en el Gráfico 3 se presenta el diagrama de caja y bigotes de la distribución del conjunto de datos de números y geometría.

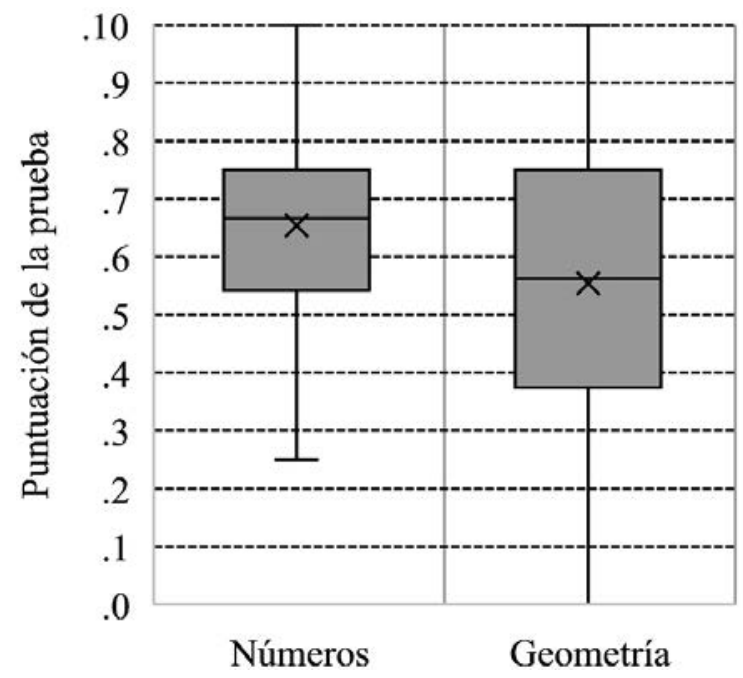

Gráfica 3. Puntuaciones según dominio de contenido (números y geometría)

Nota: Fuente propia de la investigación.

En el dominio de números, el $50 \%$ de estudiantes obtiene notas inferiores a 6.67. En el dominio de geometría, la mediana es de 5.63 , lo cual indica que el $50 \%$ de estudiantes tiene notas inferiores a ese valor. En el dominio de números se ve una distribución asimétrica negativa y en el dominio de geometría tiene una distribución simétrica (es decir, los datos se distribuyen de forma uniforme).

La Tabla 3 muestra la media y desviación estándar de las puntuaciones obtenidas en los dominios de números y geometría, respectivamente. Además, se ha realizado un test estadístico para analizar si la diferencia entre las medias es significativa.

Tabla 3. Estadísticos de números y geometría

\begin{tabular}{lcc}
\hline & Números & Geometría \\
\hline Media & 6.54 & 5.54 \\
Desviación & 1.65 & 2.57 \\
p-valor & \multicolumn{2}{c}{0.00153} \\
\hline Nota: Fuente propia de la investigación.
\end{tabular}

A partir de los resultados que se muestran en la Tabla $3(p=0.00153)$ se rechaza la hipótesis nula (Ho) planteada en H4. Así, existen diferencias significativas entre los resultados de la prueba de números y geometría.

Dominio cognitivo (H4). En este apartado se analizan los resultados obtenidos según el dominio cognitivo: conocer, aplicar y razonar. El Gráfico 4 muestra los valores de la media, mediana y cuartiles obtenidos por el estudiantado en la prueba por dominio cognitivo.

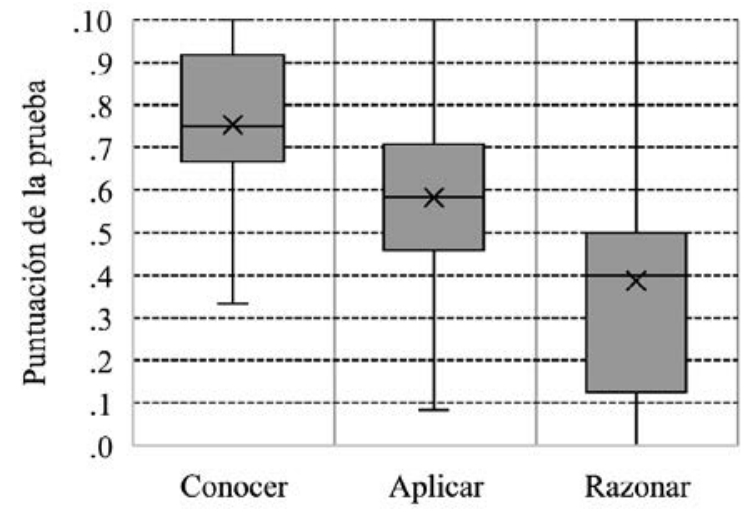

Gráfica 4. Puntuaciones según dominio cognitivo (conocer, aplicar y razonar) Nota: Fuente propia de la investigación.

En conocimiento, el $50 \%$ de estudiantes tiene notas inferiores a 7.50, en aplicar a 5.83 y en razonamiento a 4.00 .

Para comprobar la hipótesis de la homogeneidad de los grupos de dominio 
cognitivo de la prueba aplicada, se utiliza el análisis de varianza (ANOVA). La prueba de ANOVA da como resultado $\mathrm{F}=56.6$; $\mathrm{p}$ $<0.001$, lo que implica que hay diferencias estadísticamente significativas en los grupos de estudio. Seguidamente, se verifica en qué grupos existe esa diferencia. Para esto se aplica la prueba estadística HSD (Honestly Significant Difference) de Tukey. La prueba HSD muestra que existe diferencias significativas entre: aplicación y conocimiento $(\mathrm{p}$ $<0.01$ ), razonamiento y conocimiento $(\mathrm{p}<$ $0.01)$, y razonamiento y aplicación $(\mathrm{p}<0.01)$.

\section{DISCUSIÓN Y CONCLUSIONES}

En esta investigación los resultados se presentaron en dos secciones. En la primera parte, como primer punto, se analizaron los resultados por tipo de bachillerato (H1). Se ha evidenciado que hay diferencias significativas entre los dos grupos. Estudiantes de primer año del Grado de Educación Primaria que provienen de un bachillerato de Ciencias obtienen una media mayor que quienes estudiaron el bachillerato de Humanidades y Ciencias Sociales. Este resultado corrobora lo dicho por Nortes y Martínez (1992) y Ruiz de Gauna et ál. (2013), quienes encontraron que estudiantes provenientes del bachillerato de Ciencias obtuvieron mejores resultados en las pruebas aplicadas de matemáticas y son los grupos más motivados. Seguidamente, se verificó la hipótesis 2: estudiantes que han tenido la asignatura de matemáticas en los dos últimos años obtienen una media superior a quienes no han tenido matemáticas en los dos últimos años. El análisis estadístico ha mostrado que la diferencia entre ambas medias es estadísticamente significativa.
En la segunda parte, se estudia el dominio de contenido matemáticos y dominio cognitivo. Respondiendo la H3, se evidencia una diferencia estadísticamente significativa entre la media de las puntuaciones que se obtiene en el dominio de números y en el de geometría. Coincidimos con Nortes y Nortes (2017a) en que geometría es el bloque con peores resultados. Por otro lado, en el dominio cognitivo, existen diferencias significativas en los tres grupos planteados en la prueba, por tanto se rechaza la hipótesis nula (Ho) planteada en H4. Se observa que el estudiantado tiene dificultades en desarrollar los problemas encaminados a la aplicación y al razonamiento (evaluación por destrezas). Estos resultados son consistentes con los presentados por Bocco y Canter (2010) en donde se evidenció que el estudiantado tiene grandes dificultades para resolver situaciones problemáticas de la vida real.

En síntesis, en este estudio se realiza un análisis estadístico para cada hipótesis planteada. Los resultados muestran que hay diferencias estadísticamente significativas entre estudiantes de primer año del Grado de Educación Primaria que cursaron el bachillerato de Ciencias y Humanidades y Ciencias Sociales, pues estudiantes que provienen del bachillerato de Ciencias obtienen puntuaciones más altas. En esta investigación, también, se mostró que estudiantes de primer curso del Grado de Educación Primaria que no han estudiado matemáticas en los dos últimos años obtienen puntuaciones inferiores a quienes han estudiado en los últimos dos años. Es evidente que estudiantes que no han estudiado en los últimos años matemáticas obtienen puntuaciones inferiores, estos resultados también pueden estar estrechamente relacionados con $\mathrm{H} 1$, ya que no todo el estudiantado que 
proviene de un bachillerato de Humanidad y Ciencias Sociales estudió matemática en los últimos años, debido a que la asignatura no es obligatoria.

Otro resultado importante que se puede resaltar en esta investigación es que el estudiantado muestra mejor conocimiento en el tema de números (aritmética), y dificultades en el desarrollo de problemas de geometría. También, es importante remarcar que en el análisis del dominio cognitivo: conocer, aplicar y razonar, estudiantes de docencia tuvieron mejor desempeño en la faceta cognitiva de conocimientos y se les dificulta responder las preguntas de los aspectos cognitivos de aplicar y razonar.

Una de las claves para poder mejorar la calidad de la formación de maestros y maestras sería que todo el estudiantado que ingresa en el Grado de Educación Primaria entendiera los conocimientos matemáticos básicos que se aprenden en primaria y secundaria. En algunos casos, quizás los han olvidado, pero en la mayoría, el problema es que quizás no los entendieron en su momento. Por ello, se propone generar un curso inicial de conocimientos matemáticos para estudiantes que no han tenido matemáticas en los dos últimos años y estudiantes que presenten debilidades en el aprendizaje de números y geometría. Este curso tendría dos bloques temáticos: el de números y el de geometría. En este se pretenderá reforzar sus capacidades cognitivas de analizar y razonar. Como dice Alguacil et ál. (2016), la finalidad principal del profesorado es ayudar a sus estudiantes a desarrollar el razonamiento matemático, su capacidad de formular y resolver problemas.

También, es necesario que los centros de educación superior exijan una prueba de aptitudes para el ingreso a la carrera de maestro o maestra, con el propósito de tener estudiantes en primer año con las competencias básicas asumidas. En la comunidad de Cataluña, desde el 2017, estudiantes que van a ingresar en el Grado de Educación Primaria o en el Grado de Educación Infantil tienen que superar una prueba de aptitudes personales (PAP).

Además, se tendrían que reforzar los contenidos que se enseñan en las asignaturas de enseñanza-aprendizaje de las Matemáticas del Grado de Educación Primaria, tomando en consideración las deficiencias que presentan en los temas de geometría. Sin embargo, este estudio aporta resultados que nos indican que debemos reforzar el dominio cognitivo de razonamiento.

Finalmente, es importante destacar que esta investigación puede ser de utilidad, tanto para cualquier universidad que se dedique a la formación inicial de docentes, como para las escuelas e institutos. El propósito es mejorar la calidad de la enseñanza y el aprendizaje de las matemáticas. Y para ello, es indispensable conocer las carencias que presenta el estudiantado. En muchos casos, se trata de contenidos o procedimientos que se enseñaron durante la etapa de Educación Primaria. Los resultados evidencian que no se comprendieron correctamente.

En una futura investigación se volverá a aplicar la misma prueba cuando el estudiantado participante esté en un curso superior, para verificar, si su conocimiento de contenido matemático ha tenido algún tipo de variación, una vez hayan recibido formación de matemáticas.

\section{RECONOCIMIENTOS}

Este trabajo es apoyado por el programa Martí i Franquès Predoctoral Research Grant 2017PMF-PIPF-41, de la Universitat Rovira i Virgili 


\section{DECLARACIÓN DE LA CONTRIBUCIÓN DE LOS AUTORES}

El porcentaje total de contribución para la conceptualización, preparación y corrección de este artículo fue el siguiente: J.S. $60 \%$, C.J. $40 \%$.

\section{DECLARACIÓN DE DISPO- NIBILIDAD DE LOS DATOS}

Los datos que respaldan los resultados de este estudio serán puestos a disposición por el autor correspondiente [J.S.], previa solicitud razonable.

\section{REFERENCIAS}

Alguacil, M., Boqué, M. y Pañellas, M. (2016). Dificultades en conceptos matemáticos básicos de los estudiantes para maestro. International Journal of Developmental and Educational Psychology (Revista INFAD de Psicología), 1(1), 419-430. https://doi.org/10.17060/ijodaep.2016.n1.v1.162

Alpízar-Vargas, M., \& Alfaro-Arce, A. (2019). College education of elementary school teachers: the case of mathematics. Uniciencia, 33(2), 110-154. doi: https://doi.org/10.15359/ ru. $33-2.8$

Ball, D., Lubienski, S., \& Mewborn, D. (2001). Research on teaching mathematics: The unsolved problem of teachers' mathematical knowledge. Handbook of research on teaching, 4, 433-456.

Bocco, M. y Canter, C. (2010). Errores en geometría: Clasificación e incidencia en un curso preuniversitario. Revista Iberoamericana de Educación, 53(2), 1-13. https://doi.org/10.35362/ rie5321742

Castro, A., Gorgorió, N. y Prat, N. (2015). Investigación en Educación Matemática XIX. En C. Fernández, M. Molina y N. Planas (Eds.), Conocimiento matemático fundamental en el Grado de Educación Primaria: Sistema de numeración decimal y valor posicional (221228). SEIEM.

Flores, A. R., Alfaro, M. P., González, J. E. y Rojas, N. (2018). El conocimiento especializado de un profesor de matemáticas: Un estudio de caso sobre la enseñanza de los conceptos básicos de función. Uniciencia, 32(1), 89-107. doi: 10.15359/ru.32-1.6

Giaconi, V., Perdomo-Díaz, J., Cerda, G. y Saadati, F. (2018). Prácticas docentes, autoeficacia y valor en relación con la resolución de problemas de matemáticas: diseño y validación de un cuestionario. Enseñanza de las ciencias, 36(3), 99-120. doi: 10.5565/rev/ ensciencias. 2351

Gutiérrez, A., Gómez, P. y Rico, L. (2016). Conocimiento matemático sobre números y operaciones de los estudiantes de Magisterio. Educación XXI, 19(1), 135-158. https://doi. org/10.5944/educxx1.15581

Haciomeroglu, G. (2006). Prospective secondary teachers' subject matter knowledge and pedagogical content knowledge of the concept of function [Tesis doctoral. The Florida State University College of Education].

Hernández, R., Fernández, C. y Baptista, P. (2007). Metodología de la investigación (4. ${ }^{\circ} \mathrm{ed}$ ). McGraw-Hill.

Instituto Nacional de Evaluación Educativa (INEE). (2012). TIMSS 2011. Preguntas liberadas de matemáticas $-8^{\circ}$ grado. https://bit.ly/2FpHjmt

Ma, L. (2010). Conocimiento y enseñanza de las matemáticas elementales: la comprensión de las matemáticas fundamentales que tienen los profesores en China y los EE.UU. Academia Chilena de Ciencias.

Mahler, D., Großschedl, J., Harms, U. (2017). Using doubly latent multilevel analysis to elucidate relationships between science teachers' professional knowledge and students' performance. International Journal of Science Education, 39(2), 213-237. doi: 10.1080/09500693.2016.1276641

Martín del Pozo, R., Fernández, P., González, M. \& de Juana, A. (2013). El dominio de los contenidos escolares: Competencia profesional y formación inicial de maestros. Revista de Educación, 360, 363-287.

Martín, M. y Puertas, R. (2018). Comparativa de la eficiencia educativa de Europa y Asia: TIMMS 2015=Comparison of educational 
efficiency in Europe and Asia: TIMMS 2015. Revista de Educación, 380, 45-74.

Muñoz, G., Rodríguez, P. y Luque, M. (2019). La formación inicial del profesorado de educación secundaria en España: Perfil y motivaciones del futuro docente. Educación XX1, 22(1), 71-92. doi: 10.5944/educxx1.20007

Nortes, A. y Martínez, R. (1992). Aptitud, actitud y rendimiento en matemáticas: Un estudio en primero de magisterio. Suma, 10, 36-40.

Nortes, R. y Nortes, A. (2017a). Competencia matemática, actitud y ansiedad hacia las matemáticas en futuros maestros. Revista Electrónica Interuniversitaria de Formación del Profesorado, 20(3), 145-160. doi: 10.6018/ reifop.20.3.290841

Nortes, R. y Nortes, A. (2017b). ¿Aritmética elemental o geometría elemental? Un estudio en futuros maestros. Educatio Siglo XXI, 35(2), 209-228. doi: $10.6018 / \mathrm{j} / 298581$

Nortes, R. y Nortes, A. (2017c). Matemáticas escolares en futuros maestros: Un estudio necesario. Profesorado. Revista de Currículum y Formación de Profesorado, 21(1), 368-386.

Nortes, R. y Nortes, A. (2018). Conocimientos matemáticos de futuros maestros en resolución de problemas de $6 .{ }^{\circ}$ de primaria. Educatio Siglo XXI, 36(3), 201-230. https://doi. org $/ 10.6018 / \mathrm{j} / 349971$

Patton, M. (2002). Qualitative evaluation and research methods (3rd ed.). Sage.

Ruiz de Gauna, J., García, J. y Sarasua, J. (2013). Perspectivas de los alumnos de Grado de Educación Primaria sobre las matemáticas y su enseñanza. Números, 82, 5-15.

Shulman, L. S. (1987). Knowledge and teaching: foundations of the new reform. Harvard Educational Review, 57(1), 1-22. https://doi. org/10.17763/haer.57.1.j463w79r56455411

Torregrosa, G., Quesada, H. y Penalva, M. C. (2010). Razonamiento configural como coordinación de procesos de visualización. Enseñanza de las Ciencias, 28(3), 327-340. doi: 10.5565/ $\mathrm{rev} / \mathrm{ec} / \mathrm{v} 28 \mathrm{n} 3.187$

Turnuklu, E. \& Yesildere, S. (2007). The Pedagogical Content Knowledge in Mathematics: Pre-Service Primary Mathematics Teachers' Perspectives in Turkey. Issues in the Undergraduate Mathematics Preparation of School Teachers, 1, 1-13.

\section{(C) $(1 \otimes \Theta \Theta$}

Conocimiento matemático de estudiantes para docentes de Educación Primaria: Análisis de variables (Jaime Segarra • Carmen Julià). Uniciencia is protected by Attribution-NonCommercial-NoDerivs 3.0 Unported (CC BY-NC-ND 3.0) 


\section{ANEXO 1}

Tabla 1.1. Instrumento que se usó para evaluar en esta investigación

\begin{tabular}{|c|c|}
\hline 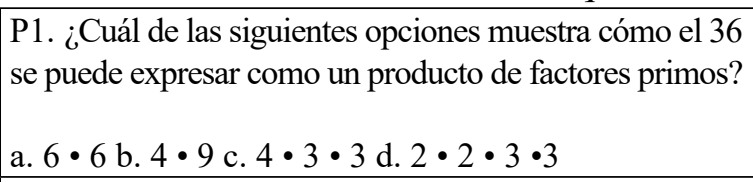 & $\begin{array}{l}\text { P2. ¿Cuál fracción es equivalente a } 0,125 ? \\
\text { a. } \frac{125}{100} \text { b. } \frac{125}{1.000} c \cdot \frac{125}{10.000} \text { d. } \frac{125}{100.000}\end{array}$ \\
\hline $\begin{array}{l}\text { P3. ¿Qué opción muestra el método correcto para } \\
\text { encontrar } \frac{1}{3}-\frac{1}{4} \text { ? } \\
\text { a. } \frac{1-1}{4-3} \text { b. } \frac{1}{4-3} \text { c. } \frac{3-4}{3 * 4} \text { d. } \frac{4-3}{4 * 3}\end{array}$ & $\begin{array}{l}\text { P4. ¿Qué número representa } \mathrm{K} \text { en esta recta numérica? } \\
\text { a. } 27.4 \text { b. } 27.8 \text { c. } 27.9 \text { d. } 28.2\end{array}$ \\
\hline $\begin{array}{l}\text { P5. ¿Qué número es igual a } \frac{3}{5} \text { ? } \\
\text { a. } 0.8 \text { b. } 0.6 \text { c. } 0.53 \text { d. } 0.35\end{array}$ & $\begin{array}{l}\text { P6. Resuelve } \frac{4}{100}+\frac{3}{1000} \\
\text { a. } 0.043 \text { b. } 0.1043 \text { c. } 0.403 \text { d. } 0.43\end{array}$ \\
\hline $\begin{array}{l}\text { P7. Las fracciones } \frac{4}{14} y_{\frac{21}{21}} \text { son equivalentes. } \\
\text { a. } 6 \text { b. } 7 \text { c. } 11 \text { d. } 14\end{array}$ & $\begin{array}{l}\text { P8. Un trabajador cortó } \frac{1}{5} \text { de cañería. El pedazo } \\
\text { que cortó mide } 3 \text { metros. ¿Cuántos metros medía la } \\
\text { cañería original? } \\
\text { a. } 8 \text { b. } 12 \text { c. } 15 \text { d. } 18\end{array}$ \\
\hline P9. Realice la siguiente operación: $42.65+5.748=$ & $\begin{array}{l}\text { P10. Ana y Jenny dividen } 560 \text { euros entre ellas. Si } \\
\text { Jenny obtiene } \frac{3}{8} \text { del dinero, ¿cuántos zeds tendrá } \\
\text { Ana? } \\
\text { Respuesta: }\end{array}$ \\
\hline $\begin{array}{l}\text { P11. Carla está envasando huevos en cajas. Cada caja } \\
\text { tiene capacidad para } 6 \text { huevos. Ella tiene } 94 \text { huevos. } \\
\text { ¿Cuál es el menor número de cajas que necesita para } \\
\text { envasar todos los huevos? } \\
\text { Respuesta: }\end{array}$ & $\begin{array}{l}\text { P12. El gráfico muestra las ventas de dos tipos de } \\
\text { bebidas gaseosas durante } 4 \text { años. Si las tendencias de } \\
\text { las ventas continúan durante los próximos } 10 \text { años, } \\
\text { determina el año en que las ventas de Guinda Cola } \\
\text { serán iguales a las ventas de Limón Cola. }\end{array}$ \\
\hline
\end{tabular}




\begin{tabular}{|c|c|}
\hline $\begin{array}{l}\text { P13. El largo del lado de cada uno de los cuadrados } \\
\text { pequeños representa } 1 \mathrm{~cm} \text {. Dibuja un triángulo isós- } \\
\text { celes con base de } 4 \mathrm{~cm} \text { y altura de } 5 \mathrm{~cm} \text {. }\end{array}$ & $\begin{array}{l}\text { P14. El siguiente dibujo muestra una figura hecha de } \\
\text { cubos del mismo tamaño. Un agujero atraviesa la figura. } \\
\text { ¿Cuántos cubos se necesitarían para llenar el agujero? } \\
\text { a. } 6 \text { b. } 12 \text { c. } 15 \text { d. } 18\end{array}$ \\
\hline $\begin{array}{l}\text { P15. El volumen de la caja rectangular es de } 200 \mathrm{~cm}^{3} \text {. } \\
\text { ¿Cuál es el valor de } \mathrm{x} \text { ? }\end{array}$ & $\begin{array}{l}\text { P16. Una hoja de papel rectangular se dobla por la } \\
\text { mitad, como se muestra en la figura de abajo. Luego se } \\
\text { corta por la línea punteada y se abre el pequeño trozo } \\
\text { recortado. ¿Cuál es la forma de la figura recortada? } \\
\text { a. Un triángulo isósceles. } \\
\text { b. Dos triángulos isósceles. } \\
\text { c. Un triángulo rectángulo. } \\
\text { d. Un triángulo equilátero. }\end{array}$ \\
\hline $\begin{array}{l}\text { P17. El perímetro de un cuadrado es } 36 \mathrm{~cm} \text {. ¿Cuál es } \\
\text { el área de este cuadrado? } \\
\text { a. } 81 \mathrm{~cm}^{2} \text { b. } 36 \mathrm{~cm}^{2} \text { c. } 24 \mathrm{~cm}^{2} \text { d. } 18 \mathrm{~cm}^{2}\end{array}$ & $\begin{array}{l}\text { P18. El área de un cuadrado es } 144 \mathrm{~cm}^{2} . \text { ¿Cuál es el } \\
\text { perímetro del cuadrado? } \\
\text { a. } 12 \mathrm{~cm} \mathrm{b.} 48 \mathrm{~cm} \text { c. } 288 \mathrm{~cm} \text { d. } 276 \mathrm{~cm}\end{array}$ \\
\hline $\begin{array}{l}\text { P19. En la siguiente figura, ¿cuál es el área en } \mathrm{cm}^{2} \mathrm{de} \\
\text { la región sombreada? } \\
\text { a. } 24 \text { b. } 44 \text { c. } 48 \text { d. } 72\end{array}$ & $\begin{array}{l}\text { P20. Raúl está empacando libros en una caja rectan- } \\
\text { gular. Todos los libros son del mismo tamaño. } \\
\text { ¿Cuál es el mayor número de libros que entrará en la caja? }\end{array}$ \\
\hline
\end{tabular}

Nota: Instituto Nacional de Evaluación Educativa (INEE, 2012). 